\title{
Muskuloskelettale Veränderungen rund um die Geburt
}

\author{
Barbara Köhler
}

\author{
Eine Schwangerschaft hat tiefgreifende Veränderungen von Muskeln, Gelenken und Nerven \\ zur Folge. Es kann zu Beschwerden auf muskuloskelettaler Ebene kommen, die noch lange \\ nach der Geburt bestehen bleiben. Physiotherapie in der Schwangerschaft und nach der \\ Geburt leistet einen wichtigen Beitrag, um Schmerzen zu reduzieren sowie eine gesunde \\ Haltung und physiologische Bewegungsabläufe wiederherzustellen.
}

\section{Hintergrund}

Eine Schwangerschaft bewirkt rasche muskuloskelettale Veränderungen. Verschiedene Schmerzsyndrome können daraus resultieren und bedürfen einer adäquaten physiotherapeutischen Behandlung. Haltungsbedingte Beschwerden, die schon vor der Schwangerschaft bestanden haben, können sich verstärken. Zudem verändern physiologische Anpassungen an die Schwangerschaft und den wachsenden Fötus den Hormonhaushalt, das Blutvolumen und die Atemkapazität.

Am offensichtlichsten ist die Weitung des Bauchraums. Bauchmuskeln und die hier angesiedelten Faszien werden sehr stark gedehnt bzw. überdehnt. Daraus resultieren für viele Frauen Rücken- oder Beckenprobleme, die häufig auch nach der Geburt bestehen.

\section{Gelenke, Muskeln und Innervation}

Schwangerschaftsbeschwerden werden häufig als „normal“ und „vorübergehend“ bezeichnet, was jedoch nicht stimmt. Auch nach der Geburt passiert es häufig, dass der Körper der Frau sich nicht automatisch wieder in den vorgeburtlichen Zustand zurückbildet. Monatelang aufgebaute Fehlhaltungen und körperliche Anpassungen an Schwerpunktverlagerungen bleiben zunächst bestehen.

Zu Beginn der Schwangerschaft quillt unter dem Einfluss des Hormons Östrogen das Knorpelgewebe auf. Relaxin bewirkt das Aufquellen des Bindegewebes. So weitet sich z. B. die Symphyse des Os pubis um 0,5-12 mm. Solch massive Veränderungen haben Einfluss auf die Propriozeption und die kortikale Steuerung. Menschen mit überbeweglichen Gelenken haben eine reduzierte Sensibilität im Vergleich mit Menschen mit normwertiger
Gelenkbeweglichkeit. Noch fehlen Langzeitstudien, die den Wirkzusammenhang zwischen Bandlaxitität in der Schwangerschaft und späterer Gelenkdegeneration untersuchen. Obwohl die Muskelspindel für die Propriozeption zuständig ist, sollte die Rolle der Gelenke nicht vernachlässigt werden. Mechanorezeptorische Reflexe und Muskelspannung sind der Grund für Muskelverspannungen. Diese Störungen werden häufig als „unbeeinflussbar“ und als „normale Schwangerschaftsbeschwerden“ eingestuft. Doch das zentrale Nervensystem wird durch jegliche Art von Veränderungen beeinflusst. Vielfältige Bewegungsmodifikationen können daraus resultieren.

\footnotetext{
Merke

Das muskuloskelettale System setzt sich aus Gelenken, Muskeln und Nerven zusammen. Jedes System hängt mit dem anderen zusammen, es gibt daher keine isolierte Schädigung.
}

Die Aufgabe der Physiotherapie ist es, diese Modifikationen des muskuloskelettalen Systems zu erkennen. Denn die Veränderungen zeigen sich nicht nur lokal, sondern im gesamten Körper. Hauptsächlich lassen sich Veränderungen im Bereich des unteren Rückens und des Beckens finden. Hierbei sollte die Unterscheidung zwischen den Symptomen und dem effektiven Zugang zum Gesundheitsproblem getroffen werden. Alle drei Bewegungssysteme müssen in ihrer Interaktion betrachtet werden.

\section{Schwangerschaft und Haltung}

In der Schwangerschaft verändert sich die Haltung der Frau. Die entstehenden Verschiebungen können auch nach der Geburt bestehen bleiben und Haltung und Bewegungsabläufe dauerhaft beeinflussen. 


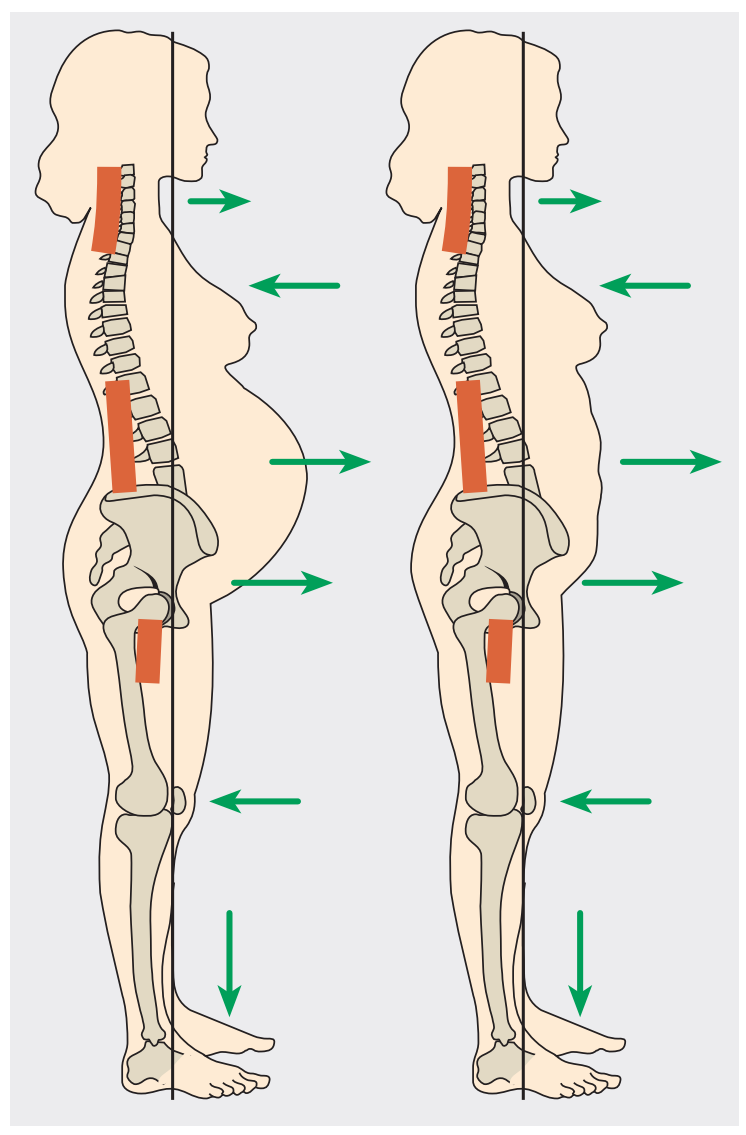

- Abb. 1 Verlagerungen des Körperschwerpunkts und verschiedener Körperabschnittsschwerpunkte (grün) und nachfolgende Verkürzungen und damit Insuffizienz der Muskulatur (rot). (Illustration: Christiane von Solodkoff)

Was unter normaler Haltung aus anatomischer und physiologischer Sicht zu verstehen ist, wurde bereits im Jahr 1943 definiert. Ein wichtiger Faktor dabei ist eine gute Balance im Stand, die auch von der Funktionsfähigkeit der Beckenbodenmuskulatur abhängt. Die normwertige Lotlinie im Stand fällt durch den Processus mastoideus, durch das Acromion, durch die Hüftgelenke, durch die Patella und durch das Sprunggelenk und teilt somit den Körper in zwei etwa gleich schwere Hälften.

In der Schwangerschaft kommt es jedoch zu einer Ventralverschiebung der Halswirbelsäule, der Lendenwirbelsäule und des Beckens. Die Kniegelenke werden überstreckt und die Fußwölbungen flachen ab. Demzufolge werden die Muskeln in den betroffenen Bereichen angenähert und passiv insuffizient. Zusätzlich erhöht sich die Belastung auf die Gelenke. Dies kann zu Ermüdungserscheinungen, Mikroverletzungen und Schmerzen in den betroffenen Körperabschnitten führen. Den stärksten Einfluss hat allerdings die Verlagerung des Körperschwerpunkts. Diese Veränderung spielt während und nach der Schwangerschaft eine große Rolle und sollte unbedingt physiotherapeutisch begleitet werden. Uneinigkeit besteht in Hinblick auf die Hyperlordose und die 
Abflachung bzw. Dorsalverschiebung des Brustkorbs. Auch die Verschiebung des Hüftgelenks mit oder ohne Ventralkippung wird in Studien unterschiedlich gewertet. Möglicherweise liegen verschiedene Haltungs- und Bewegungsmodifikationen vor.

Zusätzlich sollte neben dem Einfluss auf die Gelenke unter diesen veränderten Haltungsbedingungen auch die Funktionsfähigkeit der Lungen und der Bauchorgane bedacht werden.

\section{Wissenschaftliche Belege}

Studien zu Haltungsveränderungen in der Schwangerschaft legen divergierende Resultate vor. Die meisten Publikationen zu diesem Thema sind zwischen 1970 und 1980 erschienen. Möglicherweise spielt für die Veränderungen in der Schwangerschaft die vorher eingenommene Haltung eine wesentliche Rolle. Um das zu untersuchen, müssten prospektive Langzeitstudien durchgeführt werden, die jedoch viel Zeit und Geld in Anspruch nehmen würden.

Die in $>$ Tab. 1 aufgeführten Studien zu Haltungsveränderungen in der Schwangerschaft wurden publiziert:

\section{Haltungsveränderungen in der Schwangerschaft}

\section{Hyperlordose}

Wie beschrieben verstärkt sich die Lordose der LWS vor allem im letzten Trimester. Dumas und sein Team stellten

- Tab. 1 Zentrale historische Publikationen zu Haltungsveränderungen in der Schwangerschaft

\begin{tabular}{|l|l|}
\hline Thema & Publikationen \\
\hline Verstärkte lumbale Lordose & $\begin{array}{l}\text { Sands 1958, Spankus 1965, } \\
\text { Bullock 1987, Hummel } \\
1987 \text { [14], Bullock-Saxton }\end{array}$ \\
\hline \begin{tabular}{l} 
1991 [3], Dumas 1995 [9] \\
\hline $\begin{array}{l}\text { Anteriore Kippung des Os } \\
\text { Sacrum }\end{array}$
\end{tabular} & Sands 1958, Spankus 1965 \\
\hline $\begin{array}{l}\text { Dorsale Kippung der } \\
\text { stärkung der lumbalen } \\
\text { Lordose }\end{array}$ & $\begin{array}{l}\text { Rhodes 1958, Danforth } \\
\text { 1967, Nwuga 1982, Hum- }\end{array}$ \\
\hline $\begin{array}{l}\text { Ventraler Shift durch die } \\
\text { Vergrößerung des Uterus }\end{array}$ & Cyriax 1965 [8] \\
\hline $\begin{array}{l}\text { Überstreckung der } \\
\text { Hüftgelenke }\end{array}$ & Hummel 1987 [9] \\
\hline $\begin{array}{l}\text { Abflachung der Lendenwir- } \\
\text { belsäule und Steigerung der } \\
\text { Brustwirbelsäule im Sinne } \\
\text { einer Kyphose }\end{array}$ & $\begin{array}{l}\text { Hummel 1987, Snijders } \\
\text { [3], Hummel 1987 [14] }\end{array}$ \\
\hline
\end{tabular}

einen überraschenden Unterschied zwischen Erstgebärenden und Mehrfachgebärenden fest. Die Lordose nach der Geburt war bei Mehrfachgebärenden deutlich ausgeprägter - vermutlich wegen der höheren familiären und häuslichen Belastung [9].

\section{Schmerzen in der Brustwirbelsäule}

Neben Schmerzen in der Lendenwirbelsäule können bei Schwangeren Schmerzen im Bereich der Brustwirbelsäule auftreten [1]. Unklar ist bislang, ob es sich um Gelenkprobleme, Veränderungen des Atemmusters, Einschränkungen der Rippenbeweglichkeit oder Verspannung der mm. scaleni handelt. Uneinigkeit besteht auch darin, ob sich die Kyphose verstärkt oder nur eine Dorsalverschiebung erfolgt oder ob beides zutrifft.

\section{Spinale Instabilitäten}

Seit der Publikation von Pandjabi et al. aus dem Jahr 1992 ist bekannt, dass die Propriozeption in Bändern, Gelenkflächen und Gelenkkapseln eine bedeutende Rolle spielt. Passive Strukturen wie Wirbel, Gelenkfacetten, Bandscheiben, Gelenkbänder, Gelenkkapseln und biomechanische Einflussfaktoren gehören dazu. Eine bestehende Instabilität der Lendenwirbelsäule wird von vielen Schwangeren im letzten Trimester als verbessert beschrieben. Vermutlich ist dafür die Bewegungseinschränkung durch den raumfordernden Prozess verantwortlich [23].

\section{Therapiemöglichkeiten in der Schwangerschaft}

Zunächst sollte sichergestellt werden, dass die betroffene Frau ihren Beckenboden tatsächlich korrekt aktivieren kann und ein sinnvolles Training möglich ist. Eine Möglichkeit, die Aktivierung des Beckenbodens zu überprüfen bietet die Elektromyografie (EMG). Allerdings sind die erhobenen Daten oft ungenau, denn das Ergebnis kann durch Crosstalk angrenzender Muskeln massiv verfälscht sein. Ultraschallgestützte Verfahren und Palpation mit der Wertung nach dem Oxford-Grading können das Training und den Erfolg der Therapie wesentlich genauer dokumentieren.

Der M. transversus abdominis spielt bei der Therapie von Haltungsveränderungen eine große Rolle. Alle bekannten Übungen zur Rumpfkontrolle sind hier hilfreich. Während der Einatmung erfolgt eine Druckerhöhung im Bauchraum und der Beckenboden senkt sich. Während der Ausatmung ist der Beckenboden normalerweise aktiv und unterstützt indirekt die Ausatmung. Es gibt Berichte über die Reduktion von Schmerzen im Rücken und Beckenboden um 48-50\% bei korrektem Training [25]. 


\section{Merke}

Es ist wichtig, jede schwangere Frau aufzuklären, dass sie unter normalen Veränderungen der Schwangerschaft leidet und dass man diese therapeutisch begleiten kann. Lockerungen der Bänder und der Gelenksicherungen sind unter den hormonellen Voraussetzungen der Schwangerschaft normal. Dennoch bilden sich Veränderungen und Mikroschädigungen nach der Geburt nicht automatisch zurück und sollten ebenfalls therapeutisch begleitet werden.

\section{Situation postpartum und Therapieoptionen}

Die meisten Beschwerden flachen nach der Geburt ab, aber nicht alle. Es ist ein Trugschuss zu denken „nach einem Jahr sei alles vorbei“. Alle körperlichen und auch seelischen Veränderungen sind wichtig und sollten in die therapeutische Begleitung einbezogen werden. Haltungskorrekturen können von vielen Frauen nach der Geburt kaum durchgeführt werden, weil die Propriozeption und die Gelenk- und Muskelsituation verändert ist [9]. Zudem führen hormonelle Veränderungen in der Schwangerschaft und die Anpassungen der Wirbelsäule meist dazu, dass diese Modifikationen auch nach der Geburt bestehen bleiben.

\section{Möglichkeiten der Physiotherapie nach der Geburt}

Der wichtigste Ansatz ist die Integrierung der bewussten Haltung und Beckenbodenaktivierung in Aktivitäten des täglichen Lebens. Dazu gehören u. a. Heben, Tragen, Aufstehen, Hinsetzen, Niesen und Husten. Physiologische Bewegungsabläufe sollten so erlernt werden, dass sie in alltäglichen Situationen vor und nach der Geburt ganz selbstverständlich umgesetzt werden können. Die Physiotherapie zielt auf die Reduktion von Schmerzen, Haltungsverbesserung, Korrektur der Schultergürtelposition, Rückenstabilisation, Bauchmuskelkräftigung, Ko-Kontraktion der mm. transversus abominis und multifidii sowie Korrektur und Stabilisierung der Beinachsen ab.

\section{STUDIEN}

Physiotherapeutischen Behandlung von Haltungsveränderungen

Der Effekt einer physiotherapeutischen Behandlung bei den unter $>$ Tab. 1 aufgeführten Veränderungen wurde erstmals von Dumas et al. berichtet. Die Korrektur der Haltungsveränderungen war zwar gering, der Rückgang der Beschwerden jedoch signifikant [9]. Bullock et al. fanden zudem ein Fortbestehen der Hyperlordose der Lendenwirbelsäule nach der 
Geburt. Selbst 30 Wochen postpartum konnte diese Forschergruppe Veränderungen der Lenden- und Brustwirbelsäule bei nicht behandelten Frauen nachweisen [3].

In den Studien zeigte sich deutlich, dass jede schwangere Frau individuell behandelt werden sollte. Schwangerschaftsbedingte Probleme sind zwar ähnlich, aber nicht identisch. Zudem ist nachgewiesen, dass etwa $30 \%$ der Frauen den Beckenboden nicht korrekt aktivieren können. Auch aus diesem Grund wäre es empfehlenswert, dass alle Frauen, egal ob sie sich ein Kind wünschen oder nicht, zu einer spezialisierten Physiotherapie gehen, um die korrekte Ansteuerung der Beckenbodenmuskulatur zu erlernen.

\section{Traning in der Schwangerschaft und nach der Geburt}

\section{Vierfüßlerstand}

Geeignete Ausgangsstellungen für das Training in der Schwangerschaft sollten keinen zusätzlichen Druck auf den Bauchraum und das werdende Kind ausüben. Hier ist z. B. der Vierfüßlerstand zu empfehlen. Haltungskorrekturen in dieser Position geben dem Physiotherapeuten die Möglichkeit, die Wahrnehmung durch Handkontakt, Bewegungsaufträge und visuelle Kontrollen (Spiegel oder Foto) einzusetzen. Zudem kommt es zu einer reaktiven Ko-Kontraktion der relevanten Rumpf- und Schultermuskulatur. Sinnvolle Heimübungen lassen sich so vermitteln.

\section{Übungen im Sitzen}

Auch eine sitzende Position in verschiedenen Höhen ist als Ausgangsstellung zu empfehlen. Wenn die Hüftgelenke einen Winkel von $60 \%$ ergeben, findet die stärkste Druckübertragung auf die Sitzhöcker statt. In dieser Position muss die Frau die aktive Rumpfkontrolle am intensivsten durchführen. Tiefere Sitzpositionen bewirken einen passiven Haltungsausgleich im Sinne der Entschärfung der lumbalen Hyperlordose, was bei entsprechender Problematik sinnvoll sein kann.

\section{Übungen im Stehen}

Der Stand ist ebenfalls eine gute, aber auch anspruchsvolle Ausgangsstellung. Viele Gelenke und Muskeln sind dabei unter Kontrolle zu halten.

\section{Physiotherapeutisches Training}

Die Physiotherapie unterstützt das Training durch:

- verbales Feedback

- Spiegel- oder Fotokontrolle

- Fazilitation durch Handkontakt und Tapping der abgeschwächten Muskeln (insbesondere die Ko-
Kontraktion des m. transversus abdominis, der mm. mutifidii und der Beckenbodenmuskulatur im

Anschluss an das erfolgreich instruierte isoliere Training)

- manuelle Korrektur der Gelenkpositionen

- Mobilisation eingeschränkter Gelenke (wie z. B. der Ilioskralgelenke)

- Einleitung alltagsrelevanter Bewegungsabläufe

- Zudem spielt die Schulung des Gleichgewichts für die Reaktionsfähigkeit des Beckenbodens eine große Rolle.

\section{Merke}

Je besser die Frau die Instruktionen wahrnehmen und im täglichen selbstständigen Training einsetzen kann, desto nachhaltiger ist die Verbesserung der Beschwerden. Im Lauf der Zeit sollten sich die erlernten Bewegungsabläufe automatisieren [15].

Hilfsmittel wie Schuhe mit federnden Polster, gelegentlich stabilisierende Beckengürtel, Vaginaltampons, etc. können einen wertvollen Beitrag zur Unterstützung der physiologischen Haltung und Bewegung bieten.

In den verschiedenen Ausgangspositionen sollte die Beckenbodenmuskulatur zunächst isoliert und nach erfolgreichem Auftrainieren zusammen mit der angrenzenden Muskulatur arbeiten.

\section{STICHWORT}

Rektusdiastase

Eine besondere Bedeutung für die Therapie hat die Rektusdiastase. Neben der Muskelüberdehnung kommt es in der Schwangerschaft zur Überdehnung von Faszien, die sich je nach Alter und Bindegewebstypus unterschiedlich zurückbilden. Bauchmuskeltraining kann diesen Rückbildungsprozess deutlich unterstützen. In früheren Jahren wurde das Training der schrägen Bauchmuskulatur betont, um eine Vergrößerung der Diastase zu verhindern. Inzwischen wurde dies revidiert. Eine adäquate Therapie erfolgt über das Training des $\mathrm{m}$. rectus abdominis, wobei die beiden Muskelanteile von lateral manuell angenähert und fixiert werden.

Über die Trainingsmodalitäten für die Therapie muskuloskelettaler Veränderungen während und nach der Schwangerschaft besteht noch keine Einigung in der Fachwelt. Für Slow-Twitch-Fasern und Fast-Twitch-Fasern sollten vermutlich unterschiedliche Methoden eingesetzt werden. Die Empfehlungen für das Training des Beckenbodens und der angrenzenden Muskulatur reichen von 30-60\% der Maximalspannung (MVC) über 8-10 sek während 20-30 min täglich bis zu Maximalspannungen 6-8 Sekunden 2 min alle 2 Tage [24][26][27]. 
Im Falle von Schmerzen, Verspannungen und Haltungsschäden kommen etablierte physiotherapeutische Techniken zum Einsatz, müssen jedoch an die Situation angepasst werden (z. B. Unmöglichkeit der Ausgangsstellung Bauchlage während der Schwangerschaft). Zu diesen Techniken gehören u.a. Muskeldetonisation, Muskelkräftigung, Gelenkmobilisation, Haltungs- und Bewegungsschulung.

\section{KONTAKTADRESSEN}

Adressen von auf Beckenbodentraining und -therapie spezialisierten Physiotherapeuten finden Sie in der Schweiz unter www.pelvisuisse.ch, in Deutschland unter www.ag-ggup.de und in Österreich unter www.physioaustria.at.

\section{Relevanz der Therapie schwangerschaftsbedingter Veränderungen}

Die Ausdehnung der Bauchmuskulatur und der bindegewebigen Strukturen ist entscheidend für die zukünftige
Funktionsfähigkeit der Rumpf- und Beckenbodenkontrolle. Die Rektusdiastase spielt eine weitere Rolle. Sollte das Bindegewebe sich nicht auf eine adäquate Form zurückbilden, besteht die Gefahr einer leistenbruchähnlichen Situation. Hier sind Einklemmungssyndrome des Darms möglich. Aktuell gibt es keine Studien, die die Veränderungen in der frühen Schwangerschaft mit späteren Folgeerscheinungen erfassen würden. Hier besteht dringender Forschungsbedarf.

Zusammenfassend ist zu sagen, dass Schwangerschaften bei Frauen vorhersehbare Veränderungen hervorrufen. Adäquate Physiotherapie kann in der Schwangerschaft und nach der Geburt einen wertvollen Beitrag leisten, um Beschwerden vorzubeugen und dauerhaft zu reduzieren.

\section{Autorinnen / Autoren}

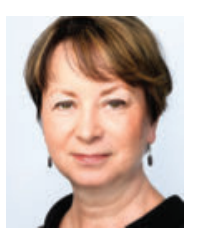

Prof. Dr. rer. medic. Barbara Köhler ist Physiotherapeutin und Gymnastiklehrerin. Sie eröffnete 2018 die Praxis für BeckenbodenGesundheit in Zürich. 


\section{Korrespondenzadresse}

Prof. Dr. Barbara Köhler

Mühlegasse 25

8001 Zürich

Schweiz

E-Mail: info@beckenboden-gesundheit.ch

\section{Literatur}

[1] Bookhout MM, Boissauld WG. Physical management of muskuloskeletal diorers during pregnancy. In: Wilder, Hrsg. Obstetric and physical therapy Clinics in Physical Therapy 1998; 20: 17-62

[2] Bullock-Saxton JE. Back pain during pregnancy. Australian Physiotherapy Association National Conference 1988: 84-91

[3] Bullock-Saxton JE. Changes in posture in standing associated with pregnancy and erly post-natal period. Physiotherapy Theory and Pracise 1991; 7(2):103-109

[4] Bullock-Saxton JE. Local sensation changes and altered hip muscle function following respetitive ankle sprain. Physical Therapy 1994; 74: 17-31

[5] Bullock-Saxton JE, Janda V, Bullock MI. Reflex activation of gluteal muscles in walking with balance shoes: an approach to restoration of function for chronic low back pain patients. Spine 1993, 18(6):704-708

[6] Calguneri M, Bird HA, Wright B Changes in joint laxity occuring in pregnancy. Annals of Rheumatic Diseases 1982; 41: 126-128

[7] Coderre T, Melzack R. Cutaneous hyperalgesia: contributions oft he peripheral and central nervous systemtot he increase in pain sensitivity after injury. Brain Research 1987; 404: 95106

[8] Cyriax J. Textbook of Orthopedic Medicine. 7. Aufl. London: Cassell; 1965

[9] Dumas GA, Reid JG, Wolfe LA. Exercise, posture and back pain during pregnancy.Clinical Biomechanics 1995. 10: 98-109

[10] Fast A, Shapiro D, Docommun EJ. Low back pain in pregnancy. Spine 1987; 12: 328-337

[11] Hainline B. Low back pain in pregnancy. In: Devinky O, Feldmann E, Hainline B, Hrsg. Neurological Complications of Pregnancy. New York: Raven Press; 1994: 65-76

[12] Henscher U. Physiotherapie in der Schwangerschaft und nach der Geburt. In: Hüter-Becker A, Dölken M, Hrsg. Physiotherapie in der Gynäkologie. Stuttgart: Thieme; 2004: 21-82

[13] Hodges PW, Richardson CA. Inefficient muscular stabilisation oft he lumbar spine associated with low back pain: a motor control evaluation aof transversus abdominis. Spine 1996; 46: 76-84

[14] Hummel P. Changes in posture during pregnancy [Dissertation], Amsterdam: Vrije Universiteit Amsterdam; 1987

[15] Janda V. Muscles, central nervorus motor regulation and back problems. In: Korr IM, Hrsg. Neurobiologic Mechanisms in Manipulative Therapy. New York: Plenum Press; 1978: 27-41

[16] Kendall HO, Kendall FP, Boynton DA. Posture and Pain. Malabar Florida: Malabar Publishing; 1952: 204

[17] Maitland GD. Vertebral manipulation. 4. Aufl. London: Butterworth; 1986

[18] Mantle M], Greenwood RM, Currey HLF. Backache in pregnancy. Rheumatology and Rehabilitation 1977; 16: 95-101

[19] Markwell S, Sapsford R. Physiotherapy management of pelvic floor dysfunction. In: Women's Health. A textbook for Physsiotherapists. London: WB Saunders Company; 1998: 383407

[20] Östgaard HC. Back pain and posterior pelvic pain in relation to pregnancy. In: Vleeming A, Mooney V, Dorman T, Snijders $C$, Hrsg. The integrated function oft he lumbar spine and sacroiliacar joint. Second Interdisziplinary World Congress on Low Back Pain: San Diego; 1995: 185-188

[21] Östgaard HC, Andersson GB. Postpartum local back pain. Spine 1992; 7(1):53-55

[22] Östgaard HC, Andersson GB, Karlsson K. Prevalence of low back pain in pregnancy. Spine 1991; 16: 549-552

[23] Pandjabi M, The stabilizing system of the spine part II. Neura zone and instability hypothesis. Journal of Spinal Disorders 1992: 5:390-397

[24] Richardson CA, Toppenberg R, Jull GA. An initial evaluation of eight abdominal exercises for their ability to provide stabilisation fort he lumbar spine. Australian Journal of Physiotherapy 1990; 36(1):6-11

[25] Rungee MJL. Low back pain during pregnancy. Orthopedics 1993; 16: 1339-1344

[26] Sapsford R, Bullock-Saxton J, Markwell S. Women's health WB Saunders, London 1999

[27] Toigo M. MuskelRevolution: Konzepte und Rezepte zum Muskelaufbau. Heidelberg: Springer Spektrum; 2015

\section{Bibliografie}

DOI https://doi.org/10.1055/a-1001-5398

Die Hebamme 2019; 32: 44-50

(c) Georg Thieme Verlag KG Stuttgart · New York

ISSN 0932-8122 